\title{
A note on spherical maxima sharing the same Lagrange multiplier
}

\author{
Biagio Ricceri*
}

Dedicated to Professor Wataru Takahashi, with esteem and friendship, on the occasion of his 70th birthday

${ }^{*}$ Correspondence:

ricceri@dmi.unict.it

Department of Mathematics,

University of Catania, Viale A. Doria

6. Catania, 95125, Italy

\begin{abstract}
In this paper, we establish a general result on spherical maxima sharing the same Lagrange multiplier of which the following is a particular consequence: Let $X$ be a real Hilbert space. For each $r>0$, let $S_{r}=\left\{x \in X:\|x\|^{2}=r\right\}$. Let $J: X \rightarrow \mathbf{R}$ be a sequentially weakly upper semicontinuous functional which is Gâteaux differentiable in $X \backslash\{0\}$. Assume that $\lim \sup _{x \rightarrow 0} \frac{J(x)}{\|x\|^{2}}=+\infty$. Then, for each $\rho>0$, there exists an open interval $I \subseteq] 0,+\infty[$ and an increasing function $\varphi: I \rightarrow] 0, \rho[$ such that, for each $\lambda \in I$, one has $\emptyset \neq\left\{x \in S_{\varphi(\lambda)}: J(x)=\sup _{S_{\varphi(\lambda)}} J\right\} \subseteq\left\{x \in X: X=\lambda J^{\prime}(x)\right\}$.
\end{abstract}

Here and in what follows, $X$ is a real Hilbert space and $J: X \rightarrow \mathbf{R}$ is a functional, with $J(0)=0$. For each $r>0$, set

$$
\begin{aligned}
& S_{r}=\left\{x \in X:\|x\|^{2}=r\right\}, \\
& B_{r}=\left\{x \in X:\|x\|^{2} \leq r\right\} .
\end{aligned}
$$

A point $\hat{x} \in S_{r}$ such that

$$
J(\hat{x})=\sup _{S_{r}} J
$$

is called a spherical maximum of $J$. Assuming that $J$ is $C^{1}$, spherical maxima are important in connection with the eigenvalue problem

$$
J^{\prime}(x)=\mu x
$$

Actually, if $\hat{x}$ is a spherical maximum of $J$, by the classical Lagrange multiplier theorem, there exists $\mu_{\hat{x}} \in \mathbf{R}$ such that

$$
J^{\prime}(\hat{x})=\mu_{\hat{x}} \hat{x}
$$

More specifically, one could be interested in the multiplicity of solutions for (1), in the sense of finding some $\mu \in \mathbf{R}$ for which there are more points $x$ satisfying (1). In this connection, however, just because of dependence of $\mu_{\hat{x}}$ on $\hat{x}$, the existence of more spherical maxima in $S_{r}$ does not imply automatically the existence of some $\mu \in \mathbf{R}$ for which (1) has more

@2014 Ricceri; licensee Springer. This is an Open Access article distributed under the terms of the Creative Commons Attribution License (http://creativecommons.org/licenses/by/2.0), which permits unrestricted use, distribution, and reproduction in any medium, provided the original work is properly cited. 
solutions. So, in order to the multiplicity of solutions of (1), it is important to know when, at least for some $r>0$, the spherical maxima in $S_{r}$ share the same Lagrange multiplier.

The aim of the present note is to give a contribution along such a direction.

Here is our basic result.

Theorem 1 For some $\rho>0$, assume that $J$ is Gâteaux differentiable in $\operatorname{int}\left(B_{\rho}\right) \backslash\{0\}$ and that

$$
\frac{\beta_{\rho}}{\rho}<\delta_{\rho}
$$

where

$$
\beta_{\rho}=\sup _{B_{\rho}} J
$$

and

$$
\delta_{\rho}=\sup _{x \in B_{\rho} \backslash\{0\}} \frac{J(x)}{\|x\|^{2}} .
$$

Assume also that, for some $a>0$, with

$$
a>\frac{\rho}{\rho \delta_{\rho}-\beta_{\rho}}
$$

if $\delta_{\rho}<+\infty$, the restriction of the functional $\|\cdot\|^{2}-a J(\cdot)$ to $B_{\rho}$ is sequentially weakly lower semicontinuous.

For each $r \in] \beta_{\rho},+\infty[$, put

$$
\eta(r)=\sup _{y \in B_{\rho}} \frac{\rho-\|y\|^{2}}{r-J(y)}
$$

and

$$
\Gamma(r)=\left\{x \in B_{\rho}: \frac{\rho-\|x\|^{2}}{r-J(x)}=\eta(r)\right\} .
$$

Then the following assertions hold:

(i) the function $\eta$ is convex and decreasing in $] \beta_{\rho},+\infty$ [, with $\lim _{r \rightarrow+\infty} \eta(r)=0$;

(ii) for each $r \in] \beta_{\rho}+\frac{\rho}{a}, \rho \delta_{\rho}[$, the set $\Gamma(r)$ is non-empty and, for every $\hat{x} \in \Gamma(r)$, one has

$$
0<\|\hat{x}\|^{2}<\rho
$$

and

$$
\begin{aligned}
\hat{x} & \in\left\{x \in S_{\|\hat{x}\|^{2}}: J(x)=\sup _{S_{\|\hat{x}\|^{2}}}\right\} \\
& \subseteq\left\{x \in \operatorname{int}\left(B_{\rho}\right):\|x\|^{2}-\eta(r) J(x)=\inf _{y \in B_{\rho}}\left(\|y\|^{2}-\eta(r) J(y)\right)\right\} \\
& \subseteq\left\{x \in X: x=\frac{\eta(r)}{2} J^{\prime}(x)\right\} ;
\end{aligned}
$$


(iii) for each $\left.r_{1}, r_{2} \in\right] \beta_{\rho}+\frac{\rho}{a}, \rho \delta_{\rho}\left[\right.$, with $r_{1}<r_{2}$, and each $\hat{x} \in \Gamma\left(r_{1}\right), \hat{y} \in \Gamma\left(r_{2}\right)$, one has

$$
\|\hat{y}\|<\|\hat{x}\|
$$

(iv) if $A$ denotes the set of all $r \in] \beta_{\rho}+\frac{\rho}{a}, \rho \delta_{\rho}$ [ such that $\Gamma(r)$ is a singleton, then the function $r \rightarrow \Gamma(r)(r \in A)$ is continuous with respect to the weak topology; if, in addition, $J$ is sequentially weakly upper semicontinuous in $B_{\rho}$, then $\Gamma_{\mid A}$ is continuous with respect to the strong topology.

Before proving Theorem 1, let us recall a proposition from [1] that will be used in the proof.

Proposition 1 Let $Y$ be a non-empty set, $f, g: Y \rightarrow \mathbf{R}$ two functions, and $a, b$ two real numbers, with $a<b$. Let $y_{a}$ be a global minimum of the function $f+a g$ and $y_{b}$ a global minimum of the function $f+b g$.

Then one has $g\left(y_{b}\right) \leq g\left(y_{a}\right)$.

Proof of Theorem 1 By definition, the function $\eta$ is the upper envelope of a family of functions which are decreasing and convex in $] \beta_{\rho},+\infty[$. So, $\eta$ is convex and non-increasing. We also have

$$
\eta(r) \leq \frac{\rho}{r-\beta_{\rho}}
$$

for all $r>\beta_{\rho}$ and so

$$
\lim _{r \rightarrow+\infty} \eta(r)=0
$$

In turn, this implies that $\eta$ is decreasing as it never vanishes. Now, fix $r \in] \beta_{\rho}+\frac{\rho}{a}, \rho \delta_{\rho}[$. So, we have

$$
\frac{\rho}{r-\beta_{\rho}}<a \text {. }
$$

Consequently, by (3),

$$
\eta(r)<a
$$

Observe that, for each $\lambda \in] 0, a\left[\right.$, the restriction to $B_{\rho}$ of the functional $\|\cdot\|^{2}-\lambda J(\cdot)$ is sequentially weakly lower semicontinuous. In this connection, it is enough to notice that

$$
\frac{a}{a-\lambda}\left(\|x\|^{2}-\lambda J(x)\right)=\|x\|^{2}+\frac{\lambda}{a-\lambda}\left(\|x\|^{2}-a J(x)\right) .
$$

Fix a sequence $\left\{x_{n}\right\}$ in $B_{\rho}$ such that

$$
\lim _{n \rightarrow \infty} \frac{\rho-\left\|x_{n}\right\|^{2}}{r-J\left(x_{n}\right)}=\eta(r) .
$$


Up to a subsequence, we can suppose that $\left\{x_{n}\right\}$ converges weakly to some $\hat{x}_{r} \in B_{\rho}$. Fix $\epsilon \in] 0, \eta(r)[$. For each $n \in \mathbf{N}$ large enough, we have

$$
\frac{\rho-\left\|x_{n}\right\|^{2}}{r-J\left(x_{n}\right)}>\eta(r)-\epsilon
$$

and so

$$
\left\|x_{n}\right\|^{2}+(\eta(r)-\epsilon)\left(r-J\left(x_{n}\right)\right)<\rho .
$$

But then, by sequential weak lower semicontinuity, we have

$$
\left\|\hat{x}_{r}\right\|^{2}+(\eta(r)-\epsilon)\left(r-J\left(\hat{x}_{r}\right)\right) \leq \liminf _{n \rightarrow \infty}\left(\left\|x_{n}\right\|^{2}+(\eta(r)-\epsilon)\left(r-J\left(x_{n}\right)\right)\right) \leq \rho .
$$

Hence, since $\epsilon$ is arbitrary, we have

$$
\left\|\hat{x}_{r}\right\|^{2}+\eta(r)\left(r-J\left(\hat{x}_{r}\right)\right) \leq \rho
$$

and so

$$
\frac{\rho-\left\|\hat{x}_{r}\right\|^{2}}{r-J\left(\hat{x}_{r}\right)}=\eta(r)
$$

that is, $\hat{x}_{r} \in \Gamma(r)$. Now, let $\hat{x}$ be any point of $\Gamma(r)$. Let us show that $\hat{x} \neq 0$. Indeed, since $\frac{r}{\rho}<\delta_{\rho}$, there exists $\tilde{x} \in B_{\rho} \backslash\{0\}$ such that

$$
\frac{J(\tilde{x})}{\|\tilde{x}\|^{2}}>\frac{r}{\rho} .
$$

Clearly, this is equivalent to

$$
\frac{\rho}{r}<\frac{\rho-\|\tilde{x}\|^{2}}{r-J(\tilde{x})}
$$

So

$$
\frac{\rho}{r}<\frac{\rho-\|\hat{x}\|^{2}}{r-J(\hat{x})}
$$

and hence, since $J(0)=0$, we have $\hat{x} \neq 0$, as claimed. Clearly, $\|\hat{x}\|^{2}<\rho$ as $\eta(r)>0$. Moreover, if $x \in S_{\|\hat{x}\|^{2}}$, we have

$$
\frac{1}{r-J(x)} \leq \frac{1}{r-J(\hat{x})}
$$

from which we get

$$
J(\hat{x})=\sup _{S_{\|\hat{x}\|^{2}}} J
$$


Now, let $u$ be any global maximum of $J_{\mid S_{\|\hat{x}\|^{2}}}$. Then we have

$$
\frac{\rho-\|u\|^{2}}{r-J(u)}=\eta(r)
$$

and so

$$
\|u\|^{2}-\eta(r) J(u)=\rho-r \eta(r) \leq\|x\|^{2}-\eta(r) J(x)
$$

for all $x \in B_{\rho}$. Hence, as $\|u\|^{2}<\rho$, the point $u$ is a local minimum of the functional $\|\cdot\|^{2}-$ $\eta(r) J(\cdot)$. Consequently, we have

$$
u=\frac{\eta(r)}{2} J^{\prime}(u)
$$

and the proof of (ii) is complete. To prove (iii), observe that

$$
\frac{1}{\eta(r)}=\inf _{\|x\|^{2}<\rho} \frac{r-J(x)}{\rho-\|x\|^{2}} .
$$

As a consequence, for each $\left.r_{1}, r_{2} \in\right] \beta_{\rho}+\frac{\rho}{a}, \rho \delta_{\rho}$ [, with $r_{1}<r_{2}$, and for each $\hat{x} \in \Gamma\left(r_{1}\right)$, $\hat{y} \in \Gamma\left(r_{2}\right)$, we have

$$
\frac{r_{1}-J(\hat{x})}{\rho-\|\hat{x}\|^{2}}=\inf _{\|x\|^{2}<\rho} \frac{r_{1}-J(x)}{\rho-\|x\|^{2}}
$$

and

$$
\frac{r_{2}-J(\hat{y})}{\rho-\|\hat{y}\|^{2}}=\inf _{\|x\|^{2}<\rho} \frac{r_{2}-J(x)}{\rho-\|x\|^{2}} .
$$

Therefore, in view of Proposition 1, we have

$$
\frac{1}{\rho-\|\hat{y}\|^{2}} \leq \frac{1}{\rho-\|\hat{x}\|^{2}}
$$

and so

$$
\|\hat{y}\| \leq\|\hat{x}\| .
$$

We claim that

$$
\|\hat{y}\|<\|\hat{x}\| .
$$

Arguing by contradiction, assume that $\|\hat{y}\|=\|\hat{x}\|$. In view of (ii), this would imply that $J(\hat{y})=J(\hat{x})$ and so, at the same time,

$$
\hat{y}=\frac{\eta\left(r_{2}\right)}{2} J^{\prime}(\hat{y})
$$

and

$$
\hat{y}=\frac{\eta\left(r_{1}\right)}{2} J^{\prime}(\hat{y}) .
$$


In turn, this would imply $\eta\left(r_{1}\right)=\eta\left(r_{2}\right)$ and hence $r_{1}=r_{2}$, a contradiction. So, (iii) holds. Finally, let us prove (iv). For each $r \in A$, continue to denote by $\Gamma(r)$ the unique point of $\Gamma(r)$. Let $r \in A$ and let $\left\{r_{k}\right\}$ be any sequence in $A$ converging to $r$. Up to a subsequence, $\left\{\Gamma\left(r_{k}\right)\right\}$ converges weakly to some $\tilde{x} \in B_{\rho}$. Moreover, for each $k \in \mathbf{N}, x \in B_{\rho}$, one has

$$
\frac{\rho-\|x\|^{2}}{r_{k}-J(x)} \leq \frac{\rho-\left\|\Gamma\left(r_{k}\right)\right\|^{2}}{r_{k}-J\left(\Gamma\left(r_{k}\right)\right)}
$$

From this, after easy manipulations, we get

$$
\begin{aligned}
& \left\|\Gamma\left(r_{k}\right)\right\|^{2}-\frac{\rho-\|x\|^{2}}{r-J(x)} J\left(\Gamma\left(r_{k}\right)\right)-\left(\frac{\rho-\|x\|^{2}}{r_{k}-J(x)}-\frac{\rho-\|x\|^{2}}{r-J(x)}\right) J\left(\Gamma\left(r_{k}\right)\right) \\
& \quad \leq \rho-\frac{\rho-\|x\|^{2}}{r_{k}-J(x)} r_{k} .
\end{aligned}
$$

Since the sequence $\left\{J\left(\Gamma\left(r_{k}\right)\right)\right\}$ is bounded above, we have

$$
\limsup _{k \rightarrow \infty}\left(\frac{\rho-\|x\|^{2}}{r_{k}-J(x)}-\frac{\rho-\|x\|^{2}}{r-J(x)}\right) J\left(\Gamma\left(r_{k}\right)\right) \leq 0
$$

On the other hand, by sequential weak semicontinuity, we also have

$$
\|\tilde{x}\|^{2}-\frac{\rho-\|x\|^{2}}{r-J(x)} J(\tilde{x}) \leq \liminf _{k \rightarrow \infty}\left(\left\|\Gamma\left(r_{k}\right)\right\|^{2}-\frac{\rho-\|x\|^{2}}{r-J(x)} J\left(\Gamma\left(r_{k}\right)\right)\right) .
$$

Now, passing in (4) to the liminf, in view of (5) and (6), we obtain

$$
\|\tilde{x}\|^{2}-\frac{\rho-\|x\|^{2}}{r-J(x)} J(\tilde{x}) \leq \rho-\frac{\rho-\|x\|^{2}}{r-J(x)} r,
$$

which is equivalent to

$$
\frac{\rho-\|x\|^{2}}{r-J(x)} \leq \frac{\rho-\|\tilde{x}\|^{2}}{r-J(\tilde{x})}
$$

Since this holds for all $x \in B_{\rho}$, we have $\tilde{x}=\Gamma(r)$. So, $\Gamma_{\mid A}$ is continuous at $r$ with respect to the weak topology. Now, assuming also that $J$ is sequentially weakly upper semicontinuous, in view of the continuity of $\eta$ in $] \beta_{\rho},+\infty[$, we have

$$
\lim _{k \rightarrow \infty} \frac{\rho-\left\|\Gamma\left(r_{k}\right)\right\|^{2}}{r_{k}-J\left(\Gamma\left(r_{k}\right)\right)}=\frac{\rho-\|\Gamma(r)\|^{2}}{r-J(\Gamma(r))}
$$

and hence

$$
\begin{aligned}
\liminf _{k \rightarrow \infty}\left(\rho-\left\|\Gamma\left(r_{k}\right)\right\|^{2}\right) & =\frac{\rho-\|\Gamma(r)\|^{2}}{r-J(\Gamma(r))} \liminf _{k \rightarrow \infty}\left(r_{k}-J\left(\Gamma\left(r_{k}\right)\right)\right) \\
& =\frac{\rho-\|\Gamma(r)\|^{2}}{r-J(\Gamma(r))}\left(r-\limsup _{k \rightarrow \infty} J\left(\Gamma\left(r_{k}\right)\right)\right) \\
& \geq \frac{\rho-\|\Gamma(r)\|^{2}}{r-J(\Gamma(r))}(r-J(\Gamma(r)))=\rho-\|\Gamma(r)\|^{2}
\end{aligned}
$$


from which

$$
\limsup _{k \rightarrow \infty}\left\|\Gamma\left(r_{k}\right)\right\| \leq\|\Gamma(r)\| .
$$

Since $X$ is a Hilbert space and $\left\{\Gamma\left(r_{k}\right)\right\}$ converges weakly to $\Gamma(r)$, this implies that

$$
\lim _{k \rightarrow \infty}\left\|\Gamma\left(r_{k}\right)-\Gamma(r)\right\|=0,
$$

which shows the continuity of $\Gamma_{\mid A}$ at $r$ in the strong topology.

Remark 1 Clearly, when $J$ is sequentially weakly upper semicontinuous in $B_{\rho}$, the assertions of Theorem 1 hold in the whole interval $] \beta_{\rho}, \rho \delta_{\rho}[$, since $a$ can be any positive number.

Remark 2 The simplest way to satisfy condition (2) is, of course, to assume that

$$
\limsup _{x \rightarrow 0} \frac{J(x)}{\|x\|^{2}}=+\infty
$$

Another reasonable way is provided by the following proposition.

Proposition 2 For some $s>0$, assume that $J$ is Gâteaux differentiable in $B_{s} \backslash\{0\}$ and that there exists a global maximum $\hat{x}$ of $J_{\mid B_{s}}$ such that

$$
\left\langle J^{\prime}(\hat{x}), \hat{x}\right\rangle<2 J(\hat{x}) .
$$

Then (2) holds with $\rho=\|\hat{x}\|^{2}$.

Proof For each $t \in] 0,1]$, set

$$
\omega(t)=\frac{J(t \hat{x})}{\|t \hat{x}\|^{2}} .
$$

Clearly, $\omega$ is derivable in $] 0,1]$. In particular, one has

$$
\omega^{\prime}(1)=\frac{\left\langle J^{\prime}(\hat{x}), \hat{x}\right\rangle-2 J(\hat{x})}{\|\hat{x}\|^{2}} .
$$

So, by assumption, $\omega^{\prime}(1)<0$ and hence, in a left neighborhood of 1 , we have

$$
\omega(t)>\omega(1)
$$

which implies the validity of (2) with $\rho=\|\hat{x}\|^{2}$.

Also, notice the following consequence of Theorem 1 .

Theorem 2 For some $\rho>0$, let the assumptions of Theorem 1 be satisfied.

Then there exists an open interval $I \subseteq] 0,+\infty[$ and an increasing function $\varphi: I \rightarrow] 0, \rho[$ such that, for each $\lambda \in I$, one has

$$
\emptyset \neq\left\{x \in S_{\varphi(\lambda)}: J(x)=\sup _{S_{\varphi(\lambda)} J}\right\} \subseteq\left\{x \in X: x=\lambda J^{\prime}(x)\right\} .
$$


Proof Take

$$
I=\frac{1}{2} \eta(] \beta_{\rho}+\frac{\rho}{a}, \rho \delta_{\rho}[) .
$$

Clearly, $I$ is an open interval since $\eta$ is continuous and decreasing. Now, for each $r \in] \beta_{\rho}+$ $\frac{\rho}{a}, \rho \delta_{\rho}\left[\right.$, pick $v_{r} \in \Gamma(r)$. Finally, set

$$
\varphi(\lambda)=\left\|v_{\eta^{-1}(2 \lambda)}\right\|^{2}
$$

for all $\lambda \in I$. Taking (iii) into account, we then realize that the function $\varphi$ (whose range is contained in $] 0, \rho[$ ) is the composition of two decreasing functions, and so it is increasing. Clearly, the conclusion follows directly from (ii).

We conclude deriving from Theorem 1 the following multiplicity result.

Theorem 3 For some $\rho>0$, assume that $J$ is sequentially weakly upper semicontinuous in $B_{\rho}$, Gâteaux differentiable in $\operatorname{int}\left(B_{\rho}\right) \backslash\{0\}$ and satisfies (2). Moreover, assume that there exists $\tilde{\rho}$ satisfying

$$
\inf _{x \in D}\|x\|^{2}<\tilde{\rho}<\sup _{x \in D}\|x\|^{2},
$$

where

$$
D=\bigcup_{r \in] \beta_{\rho}, \rho \delta_{\rho}[} \Gamma(r)
$$

such that $J_{\mid S_{\tilde{\rho}}}$ has either two global maxima or a global maximum at which $J^{\prime}$ vanishes.

Then there exists $\tilde{\lambda}>0$ such that the equation

$$
x=\tilde{\lambda} J^{\prime}(x)
$$

has at least two non-zero solutions which are global minima of the restriction of the functional $\frac{1}{2}\|\cdot\|^{2}-\tilde{\lambda} J(\cdot)$ to $\operatorname{int}\left(B_{\rho}\right)$.

Proof For each $r \in] \beta_{\rho}, \rho \delta_{\rho}$ [, in view of (7), we can pick $v_{r} \in \Gamma(r)$ (recall Remark 1$)$, so that

$$
\inf _{] \beta_{\rho}, \rho \delta_{\rho}[} \psi<\tilde{\rho}<\sup _{] \beta_{\rho}, \rho \delta_{\rho}[} \psi,
$$

where

$$
\psi(r)=\left\|v_{r}\right\|^{2} .
$$

Two cases can occur. First, assume that $\tilde{\rho} \in \psi(] \beta_{\rho}, \rho \delta_{\rho}[)$. So, $\psi(\tilde{r})=\tilde{\rho}$ for some $\tilde{r} \in$ ]$\beta_{\rho}, \rho \delta_{\rho}$ [. So, by (ii), for each global maximum $u$ of $J_{\mid S_{\tilde{\rho}}}$, we have $J^{\prime}(u) \neq 0$. As a consequence, in this case, $J_{\mid S_{\tilde{\rho}}}$ has at least two global maxima which, by (ii) again, satisfies the conclusion 
with $\tilde{\lambda}=\frac{1}{2} \eta(\tilde{r})$. Now, suppose that $\tilde{\rho} \notin \psi(] \beta_{\rho}, \rho \delta_{\rho}[)$. In this case, in view of (8), the function $\psi$ is discontinuous and hence, in view of (iv), there exists some $\left.r^{*} \in\right] \beta_{\rho}, \rho \delta_{\rho}[$ such that $\Gamma\left(r^{*}\right)$ has at least two elements which, by (ii), satisfy the conclusion with $\tilde{\lambda}=\frac{1}{2} \eta\left(r^{*}\right)$.

\section{Competing interests}

The author declares that he has no competing interests.

Received: 14 October 2013 Accepted: 10 January 2014 Published: 31 Jan 2014

\section{References}

1. Ricceri, B: Uniqueness properties of functionals with Lipschitzian derivative. Port. Math. 63, 393-400 (2006)

10.1186/1687-1812-2014-25

Cite this article as: Ricceri: A note on spherical maxima sharing the same Lagrange multiplier. Fixed Point Theory and Applications 2014, 2014:25

Submit your manuscript to a SpringerOpen ${ }^{\circ}$ journal and benefit from:

- Convenient online submission

- Rigorous peer review

- Immediate publication on acceptance

- Open access: articles freely available online

- High visibility within the field

- Retaining the copyright to your article 